\title{
LES COMPTEURS PROPORTIONNELS ET LEUR UTILISATION EN RADIOPROTECTION
}

\author{
DEUXIËME PARTIE
}

\section{3 - UTILISATION DES COMPTEURS PROPORTIONNELS A CIRCULATION GAZEUSE A LA DÉTECTION DES PARTICULES ALPHA ET BÊTA}

\author{
Y. MARQUE - H. JOFFRE \\ Service de Proiection contre les Radiations \\ Centre d'Etudes Nucléaires de Saclay \\ B.P. $n^{0} 2$ - (91) Gif-sur-Yvette
}

\begin{abstract}
RÉSUMÉ
Faisant suite à l'article publié dans le volume 2, $\mathrm{n}^{\circ} \mathrm{x}$ de "Radioprotection ", cette seconde partie présente les possibilités d'utilisation des compteurs proportionnels à circulation gazeuse pour la détection et le comptage des particules $\alpha$ et des électrons.

Après avoir rappelé les impératifs particuliers à ce mode d'utilisation, les auteurs passent en revue les différents paramètres qui régissent le fonctionnement du compteur : matériaux constitutifs, fenêtre, gaz de remplissage et vitesse de balayage. Les avantages sur les autres types de détecteurs, dans le domaine du rendement de détection et de la sensibilité, sont mis en évidence.

Enfin, deux modes d'application de ce type de détecteur au comptage simultané des particules alpha et bêta sont exposés : discrimination par empilement de compteurs et discrimination électronique par hauteur d'impulsion. Les données physiques et électroniques sont présentées dans chacun des cas, ainsi que les facteurs nécessaires à l'interprétation des résultaıs.
\end{abstract}

La technique des compteurs proportionnels a pu être parfaitement adaptée au problème du comptage des sources alpha ou bêta par la réalisation des compteurs proportionnels à circulation gazeuse. En effet, ces compteurs présentent les caractéristiques intéressantes suivantes, qui les rendent particulièrement adaptés à ces comptages :

- Grande stabilité de comptage;

- Possibilité de travailler avec des compteurs de grande surface utile de détection, permettant les comptages de filtres ou de résidus d'évaporation constituant des sources de grande surface; 
- Possibilité d'utiliser des fenêtres très minces, ou même des compteurs sans fenêtre, permettant des comptages de rayonnements très peu pénétrants;

- Faible mouvement propre, pouvant être réduit notablement par l'utilisation de montages différentiels.

\section{3.r. DÉTECTION ALPHA OU BÊTA}

\subsubsection{PRINCIPE DE FONCTIONNEMENT}

Le principe de fonctionnement du compteur proportionnel a été défini au paragraphe $\mathrm{r}$, et la figure 3 montre comment le compteur proportionnel peut être utilisé pour le comptage et la discrimination des sources de rayonnement alpha et bêta.

L'absorption rapide des particules alpha et des électrons nécessite l'utilisation de fenêtres minces et par conséquent fragiles, imposant ainsi une très faible surpression de gaz (en général ro mbars). Pour limiter les phénomènes de diffusion de l'atmosphère extérieure vers l'intérieur du compteur au niveau de la fenêtre, le gaz de comptage est constamment renouvelé, ce qui entraîne en outre une meilleure stabilité de comptage.

\subsubsection{RÉAlisation DES COMPTEURS :}

\section{a) Technologie générale}

Des compteurs de formes diverses ont été réalisés [I] [5] [43 à 48] : tubes à anode centrale, hémisphère à anode annulaire, compteurs plats de plus ou moins grande surface, dont l'anode est constituée par un ensemble de fils parallèle à la fenêtre du compteur. Ce dernier type de compteur est particulièrement intéressant en radioprotection, car il permet d'effectuer des comptages sur des prélèvements de grande surface. Les surfaces de fenêtre varient de $3 \mathrm{~cm}^{2}$ à $1400 \mathrm{~cm}^{2}$, les sections sont rectangulaires ou circulaires, et la hauteur du volume de comptage est comprise entre ro et $20 \mathrm{~mm}$.

Les matériaux constitutifs du corps du compteur doivent être exempts de contamination : le cuivre électrolytique et l'acier inoxydable sont couramment employés. Les compteurs cités à la référence [49] sont en matière plastique. Dans ce dernier cas, le bruit de fond alpha et bêta résiduel dû à la radioactivité des matériaux constitutifs est pratiquement éliminé, mais ils sont d'un usage délicat dans une ambiance neutronique éventuelle.

Les fils d'anode sont généralement en tungstène ou en molybdène (diamètre de quelques centièmes à quelques dixièmes de $\mathrm{mm}$ ), ou plus rarement en platine, qui présente l'avantage de pouvoir être facilement nettoyé à la flamme chaude $\left(1000^{\circ} \mathrm{C}\right)$. Cette dernière possibilité est particulièrement intéressante pour la décontamination des compteurs sans fenêtre.

On évite les fuites de charge entre la cathode et l'anode, au niveau de la fixation de cette dernière, par la mise en place de tubes de garde autour de l'anode.

Les effets de bord, qui ont pour conséquence une diminution du champ électrique à proximité immédiate des tubes de garde, sont atténués par des anodes auxiliaires, soit tubulaires, soit constituées par des fils d'un diamètre inférieur à celui de l'anode principale, et disposés coaxialement autour d'elle. Ces anodes 
auxiliaires sont portées à un potentiel intermédiaire à ceux de l'anode principale et de la cathode [so] [s I]. On obtient ainsi des compteurs qui ont un champ uniforme dans la totalité de leur volume utile de détection.

\section{b) Choix de la fenêtre}

La fenêtre est très souvent une feuille mince de mylar $\left(\mathrm{C}_{9} \mathrm{H}_{8} \mathrm{O}_{5}\right)$, sur laquelle est vaporisé un mince film de métal (environ $0,1 \mu \mathrm{m}$ d'aluminium). L'épaisseur de cette fenêtre, aussi faible que possible, est déterminée par les conditions d'emploi du compteur et les possibilités technologiques. Elle est généralement d'environ $0,9 \mathrm{mg} / \mathrm{cm}^{2}$. Il est possible d'obtenir une épaisseur de $0,2 \mathrm{mg} / \mathrm{cm}^{2}$, mais l'utilisation en est très délicate.

On peut également remplacer la fenêtre du compteur par le porte-échantillon lui-même. On obtient ainsi une détection dans un angle solide de $2 \pi \mathrm{sr}$, dont le rendement n'est pas affecté par l'épaisseur de la fenêtre ni de la lame d'air qui sépare l'échantillon du compteur dans le cas du compteur à fenêtre. Cette méthode, particulièrement intéressante pour la mesure des émetteurs alpha ou bêta de faible énergie, ne fait alors intervenir que la géométrie du porte-échantillon, l'autoabsorption dans l'échantillon lui-même et la rétrodiffusion. Lorsque l'ensemble utilisé est un ensemble manuel, nécessitant l'ouverture de la chambre pour le changement d'échantillon, il est nécessaire de laisser s'effectuer la circulation gazeuse pendant un certain temps avant que le compteur ait repris ses caractéristiques normales. Le délai de restitution des caractéristiques normales en fonction du temps d'arrêt du compteur, est représenté sur la figure 16 , pour un compteur à circulation de méthane à paroi en acier inoxydable, d'un volume intérieur de $500 \mathrm{~cm}^{3}$, de $300 \mathrm{~cm}^{2}$ de surface utile de comptage et pour un débit gazeux de $1 \mathrm{l} / \mathrm{h}$.

\section{c) Gaz de remplissage}

Le choix du gaz de remplissage est fonction de la nature de la particule à détecter, des conditions d'utilisation et des possibilités d'approvisionnement.

Dans un compteur proportionnel le nombre de paires d'ions formées dans le gaz est proportionnel à l'énergie perdue par la particule incidente dans le gaz du compteur.

On montre, en première approximation, que l'énergie moyenne nécessaire pour former une paire d'ions dans les différents gaz est indépendante de l'énergie de la particule primaire. Ceci est vérifié pour les électrons, de $7 \mathrm{keV}$ à $17 \mathrm{MeV}$. Pour les particules alpha, cette quantité décroît lentement lorsque l'énergie de la particule croît, pour l'oxygène et l'azote. Le tableau I donne, pour les particules alpha et bêta, les valeurs moyennes des énergies moyennes nécessaires pour former une paire d'ions dans les différents gaz utilisés dans les compteurs à circulation gazeuse [s2]. L'énergie moyenne nécessaire pour former une paire d'ions est fonction essentiellement du gaz ou du mélange gazeux, et la présence d'impuretés, même en faible quantité, peut entraîner de grandes variations de valeur, en particulier pour les gaz nobles (tableau 2) [53]. Pour un mélange gazeux, Hurst propose la formule [54] :

$$
\frac{\mathbf{r}}{w}=\left(\frac{\mathrm{I}}{w_{1}}-\frac{\mathbf{1}}{w_{2}}\right) \frac{p_{1}}{p_{1}+a p_{2}}+\frac{\mathbf{1}}{w_{2}}
$$


où $w, w_{1}, w_{2}$ sont les énergies moyennes nécessaires pour former une paire d'ions respectivement dans le mélange gazeux et dans chacun des gaz constitutifs, $p_{1}$ et $p_{2}$ les pressions partielles de remplissage, et « a » une constante caractéristique du mélange gazeux déterminé empiriquement. Le tableau ci-dessous donne différentes valeurs de " a » :

$\begin{array}{lcccccc}\text { mélange : } & \mathrm{N}_{2}-\mathrm{H}_{2} & \mathrm{~N}_{2}-\mathrm{Ar} & \mathrm{N}_{2}-\mathrm{O}_{2} & \mathrm{He}-\mathrm{Ar} & \mathrm{He}-\mathrm{H}_{2} \\ \text { " a " } & 0,28 & 0,53 & 1,06 & 0,75 & 3,55 \\ \text { mélange : } & \mathrm{He}-\mathrm{N}_{2} & \mathrm{He}-\mathrm{CH}_{4} & \mathrm{Ar}-\mathrm{H}_{2} & \mathrm{H}_{2}-\mathrm{CH}_{4} \\ \text { " a " : } & 8,45 & 0,68 & 0,56 & 4,03\end{array}$

Le mélange gazeux utilisé a une grande influence sur les caractéristiques des paliers, qui varient également en fonction des paramètres géométriques choisis. Les caractéristiques des paliers des compteurs à circulation sont généralement incluses dans les limites suivantes :

$$
\begin{array}{lll}
\text { Longueur : } & 400 \mathrm{~V} \text { à } 1300 \mathrm{~V} \\
\text { Pente }: & 0,2 \% \text { à } 3 \% \text { pour } 100 \mathrm{~V} .
\end{array}
$$

Les gaz utilisables peuvent être classés en deux catégories [48] : les gaz nobles et diatomiques (argon, hélium, azote), et les gaz polyatomiques (méthane, gaz carbonique, butane, etc.).

Les gaz de la première catégorie, argon et hélium en particulier, présentent l'inconvénient d'avoir un coefficient d'amplification qui varie très rapidement avec la haute tension, aux faibles pressions d'utilisation des compteurs à circulation gazeuse, ce qui amoindrit les qualités de stabilité du compteur. De plus, le rayonnement électromagnétique de désexcitation atomique consécutif aux ionisations dans ces gaz est susceptible de produire des photoélectrons qui perturbent la proportionnalité du détecteur. Enfin, l'argon présente un état d'excitation à vie longue qui entraine la formation d'impulsions parasites. Ils sont utilisés pour les comptages alpha, où un faible coefficient d'amplification est suffisant ( 10 à 100 ). L'addition d'un faible pourcentage de gaz polyatomique $\left(\mathrm{CO}_{2}\right.$ ou $\left.\mathrm{CH}_{4}\right)$, qui absorbe le rayonnement électromagnétique de désexcitation, réduit les inconvénients cités précédemment [s]. Par exemple, les mélanges suivants peuvent être utilisés (pourcentage en volume) :

$$
\begin{aligned}
& 98,7 \% \text { hélium }-1,3 \% \text { isobutane } \\
& 9^{6} \% \text { hélium }-4 \% \text { isobutane } \\
& 9^{8} \% \text { argon }-2 \% \text { gaz carbonique. }
\end{aligned}
$$

L'ionisation des gaz de la deuxième catégorie ne conduit pas à la production de photons capables d'entraîner la formation de photoélectrons, même à des coefficients d'amplification élevés. Les grands coefficients d'amplification nécessaires au comptage bêta $\left(10^{4}\right.$ à $\left.{ }^{10^{6}}\right)$, ne peuvent être obtenus qu'avec les gaz polyatomiques ou avec des mélanges gazeux à assez forte proportion de gaz polyatomique. Les gaz polyatomiques présentent toutefois l'inconvénient d'imposer des tensions de fonctionnement nettement supérieures à celles des gaz nobles ou diatomiques. Le méthane pur est particulièrement intéressant si l'on veut effectuer un comptage alpha dans un bruit de fond bêta important [s5]. Les mélanges ci-dessous sont d'un usage courant : 


$$
\begin{aligned}
& 90 \% \text { argon - } 10 \% \text { méthane } \\
& 90 \% \text { argon - 10 } \% \text { gaz carbonique } \\
& 84 \% \text { argon - } 16 \% \text { propane. }
\end{aligned}
$$

Si la vapeur d'eau et l'oxygène peuvent être tolérés à l'état d'impuretés, les gaz présentant un caractère électronégatif accentué sont à exclure, par suite de la formation d'ions négatifs par capture d'électrons (halogènes) [s3].

L'air est utilisable pour des facteurs d'amplification n'excédant pas roo (comptages alpha), mais son usage est limité par la présence d'oxygène.

Enfin, il peut être nécessaire d'effectuer un comptage sans fenêtre en milieu très humide (échantillon humide ou liquide par exemple) : l'influence de l'eau sur les fluctuations du taux de comptage est triple [56] :

$1^{\circ}$ Capture d'électrons primaires formant des ions $\mathrm{H}_{2} \mathrm{O}^{-}$.

L'électron de charge de l'ion $\mathrm{H}_{2} \mathrm{O}^{-}$est ensuite extrait lorsque l'ion arrive dans la zone de champ élevé à proximité de l'anode. Il en résulte :

- une diminution de la hauteur de l'impulsion électronique principale collectée;

- la création d'une impulsion parasite plus faible et distincte de la première (collection des ions $\mathrm{H}_{2} \mathrm{O}^{-}$ou des électrons véhiculés par les ions $\mathrm{H}_{2} \mathrm{O}^{-}$ initialement formés).

$2^{\circ}$ Formation d'ions $\mathrm{H}_{2} \mathrm{O}^{+}$dans l'avalanche ou par échange de charge avec le gaz du compteur. Si une impulsion survient, ces ions sont susceptibles de capturer des électrons primaires et de réduire ainsi l'impulsion principale.

$3^{\circ}$ Production d'électrons secondaires lorsque les ions $\mathrm{H}_{2} \mathrm{O}^{+}$s'approchent de la cathode. Ces électrons secondaires déchargent le compteur.

Le premier inconvénient est éliminé en opérant à un seuil de discrimination suffisamment élevé. Les inconvénients 2 et 3 sont minimisés en utilisant des gaz dont le potentiel d'ionisation est inférieur à celui de l'eau.

\begin{tabular}{|c|c|c|c|c|c|c|}
\hline $\begin{array}{l}\text { Gaz } \\
\text { Potentiel }\end{array}$ & : & $\mathrm{He}$ & $\mathrm{Ar}$ & $\mathrm{CO}_{2}$ & $\mathrm{CH}_{4}$ & $\mathrm{H}_{2} \mathrm{O}$ \\
\hline d'ionisation & : & 24,46 & 15,7 & 14,4 & ${ }^{1} 3,1$ & 12,56 \\
\hline $\begin{array}{l}\text { Gaz } \\
\text { Potentiel }\end{array}$ & $\theta^{\circ}$ & $\mathrm{C}_{2} \mathrm{H}_{6}$ & $\mathrm{C}_{2} \mathrm{H}_{4}$ & $\mathrm{C}_{2} \mathrm{H}_{2}$ & $\mathrm{C}_{3} \mathrm{H}_{8}$ & $\mathrm{C}_{4} \mathrm{H}$ \\
\hline d'ionisation & : & I 1,6 & 10,8 & 11,2 & I I, 3 & 10,34 \\
\hline
\end{tabular}

Le tableau ci-dessous donne les potentiels d'ionisation des différents gaz utilisés couramment et celui de l'eau [s7] :

A un taux d'humidité de $62 \%$, les plateaux correspondant à l'éthane, au propane, et au butane coïncident encore avec ceux de ces gaz secs.

d) Vitesse de balayage

Par diffusion de l'air ambiant à travers la fenêtre mince, le coefficient d'amplification gazeuse du compteur diminue. Il est donc nécessaire d'assurer une vitesse de balayage minimale pour réduire suffisamment et même supprimer cet inconvénient [58]. L'étude de la variation de la stabilité du taux de comptage en fonction 
de la vitesse de balayage permet de vérifier ce résultat, et doit être effectuée pour chaque compteur. Lorsque cette stabilité est atteinte, on ne gagne pas à augmenter le débit gazeux. En effet, une augmentation de la vitesse de balayage du gaz accroît le taux de comptage parasite dû à la formation de charges statiques dans le compteur. A titre d'exemple pour un compteur de $600 \mathrm{~cm}^{2}$ de surface (compteur Frieseke et Hœpfner FH-407-G), le taux de comptage est stable pour des débits variant de 0,5 à $7,51 / \mathrm{h}$, ce qui correspond à des vitesses de circulation au niveau de la fenêtre de quelques $\mathrm{mm} / \mathrm{s}$ à quelques $\mathrm{cm} / \mathrm{s}$. Lors d'une utilisation après un arrêt prolongé, ou lors d'une mise en service, le dégazage des matériaux peut être accéléré en accroissant légèrement le débit. Enfin, l'utilisation d'un régulateur de débit approprié permet de réduire à son minimum la consommation de gaz.

\subsubsection{SENSIBIlltí DE DÉteCtion}

\section{a) Rendement de détection en fonction de l'énergie [59].}

Le rendement de détection en fonction de l'énergie, pour une géométrie source-compteur donnée et pour un gaz déterminé, dépend essentiellement de l'épaisseur de la fenêtre. L'influence du rayonnement rétrodiffusé est très sensible lors d'une utilisation sans fenêtre, et conduit fréquemment, pour des émetteurs d'énergie supérieure à $250 \mathrm{keV}$, à des rendements de détection supérieurs à $50 \%$. Le rendement de détection bêta est toujours supérieur à celui obtenu avec les détecteurs à scintillation, surtout dans le domaine des très basses énergies.

b) Seuil de sensibilité.

Pour un compteur de fabrication donnée, l'évaluation du seuil de sensibilité est liée à la valeur du mouvement propre et à la stabilité du compteur. Le mouvement propre par $\mathrm{cm}^{2}$ de surface de fenêtre des compteurs actuellement fabriqués est inférieur à $\mathrm{I} \mathrm{imp} / \mathrm{mn}$, dans les conditions de comptage $\beta$ et inférieur à $0,002 \mathrm{imp} / \mathrm{mn}$ dans les conditions de comptage $\alpha$. Un blindage de $10 \mathrm{~cm}$ de plomb ou d'acier et un montage en anticoïncidence permettent de descendre à des valeurs inférieures à $0,15 \mathrm{imp} / \mathrm{mn}$ dans les conditions de comptage $\beta$, pour des compteurs plats allant jusqu'à $400 \mathrm{~cm}^{2}$ de surface.

c) Sensibilité de détection aux divers points de la surface de la fenêtre [60] :

La réalisation de compteurs plats à champ très homogène permet d'obtenir une sensibilité de détection constante sur pratiquement toute la surface utile de comptage. La figure 17 montre le diagramme obtenu en déplaçant une source de ${ }^{90} \mathrm{Sr}+{ }^{90} \mathrm{Y}$ sous un compteur à circulation de méthane (compteur Berthold type LB 630I), de $20 \mathrm{~cm}$ de diamètre.

\subsection{DÉTECTION $\alpha \beta$ SIMULTANÉE}

\subsubsection{DisCRIMINATION $\alpha \beta$ PAR EMPILEMENT DE COMPTEURS [60] [61] [62]}

La superposition de deux compteurs de mesure dont les hautes tensions sont ajustées respectivement sur les paliers de fonctionnement $\alpha$ et $\beta$ peut, dans certaines conditions, permettre de compter séparément, mais simultanément, les particules $\alpha$ et $\beta$ d'une source mixte $\alpha \beta$. Le compteur inférieur, le plus proche 
de la source, détecte seulement les particules $\alpha$ par l'emploi d'une tension appropriée. Il est mécaniquement solidaire du compteur supérieur réglé en tension pour la détection $\beta$. Un compteur de barrage vient généralement coiffer l'ensemble et, associé au compteur $\beta$ par un montage en anticoïncidence ou différentiel, permet de diminuer le mouvement propre de ce dernier. La « paroi intermédiaire» qui sépare les deux compteurs doit avoir une épaisseur telle qu'elle absorbe les particules $\alpha$ d'énergie suffisante pour s'échapper du compteur inférieur, tout en réduisant à son minimum l'absorption des particules $\beta$ destinées à être captées par le second compteur. En vue de la détermination de cette épaisseur de paroi, les figures 18 a et b donnent les relations parcours-énergie des particules $\alpha$ pour les principaux gaz utilisés dans les compteurs à circulation gazeuse, pour le mylar et pour le polyéthylène constituant la paroi intermédiaire [63].

A titre d'exemple, pour une paroi intermédiaire de $2 \mathrm{mg} / \mathrm{cm}^{2}$, mise en place sur un compteur $\alpha$ à circulation de méthane de $18 \mathrm{~mm}$ d'épaisseur équipé d'une fenêtre d'entrée de $0,9 \mathrm{mg} / \mathrm{cm}^{2}$, et pour un émetteur $\alpha$ de $5,6 \mathrm{MeV}$ placé à $10 \mathrm{~mm}$ du compteur, le nombre d'impulsions comptées sur la voie $\beta$ est inférieur à la fluctuation statistique du mouvement propre du compteur. Dans les mêmes conditions expérimentales, le rendement de détection $\beta$ pour des énergies de $\mathrm{I} s \mathrm{o}$ et $500 \mathrm{keV}$ est inférieur d'environ $30 \%$ et $s \%$ à ce qu'il serait pour un compteur simple équipé d'une fenêtre d'entrée de $0,9 \mathrm{mg} / \mathrm{cm}^{2}$.

La mise en œuvre d'une paroi intermédiaire de $2 \mathrm{mg} / \mathrm{cm}^{2}$ nécessite une régulation très poussée du débit gazeux, de façon à éliminer les déformations anormales de cette paroi, et sa détérioration par suite d'un contact avec l'électrode collectrice du compteur $\alpha$.

Les avantages de cette méthode de discrimination sont la simplicité de l'électronique mise en œuvre et une excellente discrimination $\alpha \beta$. Par contre, de par la remarque taite précédemment, la tête de détection est relativement fragile, d'autant plus que la surface de fenêtre est plus grande.

\subsubsection{DisCRIMINATION ÉLECTRONIQUE ALPHA - BÊTA}

\subsubsection{Réalisation}

Il résulte des considérations développées en 3.1.2.c que les charges collectées par suite de l'ionisation du gaz, pour une valeur donnée de la haute tension, sont différentes pour les particules alpha et pour les électrons. Suivant l'énergie du rayonnement, l'épaisseur de la fenêtre et le volume du compteur, le rapport des charges peut varier de quelques unités à quelques dizaines d'unités.

Ainsi que le montre d'ailleurs la figure 1 , la région proportionnelle permet donc, dans certaines conditions, d'effectuer une discrimination des particules traversant le compteur, de par la hauteur des impulsions collectées.

La figure 19 donne, à titre d'exemple concret, les valeurs des charges maximales collectées, pour les particules alpha et bêta en fonction de la haute tension, dans un compteur à circulation d'argon-méthane de $140 \mathrm{~cm}^{2}$ de surface (compteur S.35 - Intertechnique).

Dans la solution adoptée à la référence [49] et schématisée sur la figure 20, les impulsions sortant du compteur, après une première amplification, atteignent un ensemble de deux discriminateurs montés en parallèle. 
Le seuil du premier discriminateur est ajusté pour ne laisser passer que les impulsions de forte amplitude dues aux particules alpha, qui sont ensuite comptées sur la " voie alpha ".

La hauteur du seuil du second discriminateur est réglée de façon à laisser passer les impulsions de plus faible amplitude dues aux particules béta, tout en éliminant la majeure partie des impulsions de très faible amplitude dues au bruit de fond. Les impulsions alpha, de plus forte amplitude, franchissent également ce seuil. Enfin, un circuit de blocage, monté à la suite de ce dernier ensemble, permet de ne pas compter sur la " voie bêta » les impulsions qui ont franchi simultanément les deux seuils. Les impulsions restantes sur la voie béta sont donc en principe des impulsions bêta.

Le rapport des seuils de discrimination est d'environ roo, et la tension de fonctionnement se situe sur le palier de fonctionnement béta habituel. Le dispositif de blocage entraîne un temps mort sur la voie bêta de I $\mu$ s environ par impulsion alpha.

\subsubsection{Détermination des conditions de fonctionnement}

L'utilisation de cette méthode nécessite un réglage particulièrement minutieux de la haute tension à utiliser. Les figures 21 et 22 illustrent le comportement des deux voies de mesure, lorsque l'on fait varier la haute tension, dans les deux cas où une source alpha ou une source bêta sont placées sous le compteur.

a) Source alpha (fig. 2I).

Pour des valeurs de la haute tension inférieures à $V_{1}$, l'amplitude des impulsions fournies est inférieure à la valeur du plus petit des deux seuils de discrimination. Aucune indication n'est enregistrée. Lorsque la haute tension croît et dépasse $V_{1}$, tout en restant inférieure à $V_{2}$, la hauteur des impulsions atteint et dépasse le seuil du discriminateur de la voie $\beta$. La totalité du comptage est alors enregistrée sur la voie $\beta$. A partir de $V_{2}$, les impulsions atteignent une hauteur suffisante pour franchir le seuil du discriminateur $\alpha$. Le dispositif de blocage entre alors en fonctionnement et corrélativement à l'apparition d'un comptage sur la voie $\alpha$, on enregistre une diminution du comptage sur la voie $\beta$, puis sa disparition presque totale. L'intervalle $V_{3} V_{4}$ définit la longueur du palier $\alpha$, pour lequel le taux de comptage parasite sur la voie $\beta$ est devenu négligeable. La disparition du comptage sur la voic $\beta$ serait effectivement totale si la source $\alpha$ n'était accompagnée d'aucune émission sccondaire d'électrons, ou d'impurètés émettrices $\beta$. La tension $V_{4}$ limite la région de fonctionnement en régime proportionnel.

b) Source bêta (fig. 22).

Pour les mêmes raisons qu'au paragraphe précédent, aucune indication n'est enregistrée aux faibles valeurs de la haute tension. A partir d'une certaine valeur $V_{1}$ ou $V^{\prime}{ }_{1}$ de $V$, qui est fonction de l'énergie du rayonnement $\beta$, les impulsions franchissent le seuil du discriminateur $\beta$ et commencent à être comptées sur la voie $\beta$.

Pour les énergies $\beta$ faibles (jusqu'à $400 \mathrm{keV}$ environ), le taux de comptage croît jusqu'à ce que la haute tension atteigne la valeur $V_{2}$ qui marque le début 
d'un palier $V_{2} V_{3}$, dont la longueur est fonction de l'énergie $\beta$ (fig. 22, courbe $\mathrm{r}$ ). A partir de $V_{3}$ les impulsions ont une hauteur suffisante pour franchir le discriminateur $\alpha$ et faire ainsi jouer le dispositif de coïncidence. On enregistre alors une diminution du comptage sur la voie $\beta$ compensée exactement par l'apparition d'un comptage sur la voie $\alpha$ (fig. 22, courbe 2). Le palier en trait discontinu est le palier de fonctionnement que l'on obtiendrait en mettant hors circuit le dispositif de blocage, c'est-à-dire le palier de fonctionnement des compteurs proportionnels.

Pour les énergies $\beta$ dépassant $400 \mathrm{keV}$ (fig. 22, courbes 3 et 4 ), parallèlement à l'accroissement du comptage enregistré entre $V^{\prime}{ }_{1}$ et $V^{\prime}{ }_{2}$ sur la voie $\beta$, le comptage parasite apparaît sur la voie $\alpha$ à une tension $V_{3}^{\prime}$ inférieure à la tension $V^{\prime}$ qui marque le début du palier sur la voie $\beta$.

Les perturbations enregistrées sur la voie $\alpha$ sont donc beaucoup plus importantes que dans le cas précédent.

c) Cboix de la tension de fonctionnement.

Il résulte des paragraphes précédents que la plage de fonctionnement doit être déterminée avec soin. En effet, une tension de fonctionnement trop faible entraîne l'enregistrement d'un comptage parasite sur la voie $\beta$, au détriment du comptage $\alpha$ réel, et inversement une tension de fonctionnement trop élevée entraîne l'enregistrement d'un comptage parasite sur la voie $\alpha$, au détriment du comptage $\beta$ réel.

Pour un réglage donné de la haute tension, le taux de comptage parasite sur la voie $\alpha$ est fonction croissante de l'énergie du rayonnement $\beta$ et des dimensions du compteur. Il en résulte que la discrimination $\alpha \beta$ est d'autant moins aisée que l'énergie du rayonnement $\beta$ présent est plus élevée.

La figure 23 , établie pour un compteur à circulation d'argon méthane de $135 \mathrm{~mm}$ de diamètre (compteur Intertechnique type $\mathrm{S}_{35}$ ), montre les taux de comptage obtenu avec des sources $\alpha$ (plutonium-239) et $\beta$ (carbone-14 et strontium-yttrium-90).

Pour une source contenant à la fois du plutonium-239 et du carbone-14, les courbes 2 et 3 montrent que la tension correcte à utiliser doit être comprise entre I $325 \mathrm{~V}$ et I $600 \mathrm{~V}$ pour le comptage $\alpha$ et entre I $400 \mathrm{~V}$ et I $625 \mathrm{~V}$ pour le comptage $\beta$. Une excellente discrimination $\alpha \beta$ peut donc être obtenue en choisissant la tension de fonctionnement entre I $400 \mathrm{~V}$ et $\mathrm{I} 600 \mathrm{~V}$.

Pour une source contenant à la fois du plutonium-239 et du strontiumyttrium-90 la courbe s montre qu'une tension comprise entre I 535 et I $590 \mathrm{~V}$ permet un comptage correct du rayonnement $\beta$. Par contre, le comptage $\alpha$ correct ne peut être obtenu qu'à une tension voisine de I $325 \mathrm{~V}$. Une tension voisine de $1425 \mathrm{~V}$ permettrait un taux de comptage $\alpha$ à environ $25 \%$ par excès et un taux de comptage $\beta$ à environ $25 \%$ par défaut. Ce résultat peu satisfaisant montre la difficulté d'obtenir une bonne discrimination $\alpha \beta$ lorsque l'activité d'une source $\beta$ de forte énergie est élevée devant l'activité de la source $\alpha$ (d'un facteur 70 dans le cas de la figure 23 ). Si les activités des sources $\alpha$ et $\beta$ sont du même ordre, une bonne discrimination $\alpha \beta$ peut être obtenue en choisissant la tension de fonctionnement entre I $535 \mathrm{~V}$ et i $590 \mathrm{~V}$.

En définitive si, pour un échantillon inconnu, le taux de comptage sur la voie $\alpha$ est très inférieur au taux de comptage sur la voie $\beta$, il y a lieu d'étudier le taux 
de comptage $\alpha$ en diminuant la tension de $30 \mathrm{~V}$ environ. Si la variation est nulle le taux de comptage obtenu est correct (émission $\beta$ de faible énergie). Au contraire si la diminution de comptage est importante, il y a lieu d'effectuer une correction de comptage parasite $\beta$ sur la voie $\alpha$ (émission $\beta$ de forte énergie).

Il est également nécessaire pour chaque ensemble de comptage de déterminer expérimentalement le pourcentage des impulsions géantes, constituées par empilement au niveau du compteur, et comptées sur la voie $\alpha$. Ce pourcentage se situe généralement entre un pour cent et un pour mille.

Il faut noter enfin que, pour des sources $\alpha$ épaisses, la dégradation du spectre par autoabsorption peut entraîner un comptage parasite sur la voie $\beta$, dû aux particules $\alpha$ de très faible énergie.

Le mouvement propre $\beta$ est en général réduit par l'utilisation, comme en 3.2.1. d'un compteur de barrage connecté, soit en anticoïncidence, soit en différence. Les caractéristiques électroniques doivent être particulièrement soignées. En particulier, les gains des différents étages doivent être particulièrement stabilisés.

\subsection{DOMAINE D'UTILISATION}

\subsubsection{Controles atmosphériques [64] [65]}

A surface égale, le faible encombrement des compteurs à circulation gazeuse comparativement aux détecteurs par scintillation présente un très grand intérêt pour la construction de détecteurs d'aérosols radioactifs. Le volume, et par là même le poids des blindages éventuels s'en trouvent diminués. La possibilité d'utiliser une seule tête de comptage pour la détection simultanée des émetteurs $\alpha$ et $\beta$ (compteur unique ou empilement de compteurs) accentue encore cet avantage.

De plus, dans le cas d'appareils à filtre fixe, il est possible d'utiliser des compteurs de grande surface (détection du plutonium), ce qui permet d'augmenter le débit de l'air prélevé sans accroître sensiblement l'autoabsorption dans le dépôt, et d'accroître ainsi la sensibilité de l'appareil.

Enfin, pour une distance donnée source-compteur, la position de la source sous le compteur affectant peu le rendement de détection, ce type de détecteur est particulièrement intéressant lorsque la contamination présente sur le filtre n'est pas répartie d'une façon homogène.

\subsubsection{Controle des liquides [66] [67] [68]}

L'utilisation des compteurs proportionnels à circulation gazeuse sur des appareils de contrôle en continu de la radioactivité des eaux qui concentrent à sec l'effluent à contrôler, présente les mêmes avantages que ceux mentionnés en 3.3 .1 .

De plus, les variations de température ont une incidence beaucoup plus faible sur le fonctionnement du compteur à circulation gazeuse que sur celui du photomultiplicateur.

Dans le cas d'appareils travaillant directement sur lame d'eau, l'utilisation de compteur de très grande surface $\left(700\right.$ à $\left.800 \mathrm{~cm}^{2}\right)$ permet d'accroître la sensibilité du dispositif. 


\subsubsection{Controle des surfaces [69] [70]}

De nombreux types d'appareillage de contrôle de la contamination des surfaces utilisant des compteurs à circulation gazeuse sont fabriqués.

Les dispositifs mobiles de contrôle des sols opérant avec une association de compteurs de grande surface permettent un contrôle plus rapide que les ensembles classiques (détecteurs à scintillation ou Geiger Müller), de surface utile plus faible.

L'utilisation de ce type de détecteur pour le contrôle de la contamination des vêtements de travail ou de la peau, permet, à surface égale par rapport au détecteur par scintillation, d'atteindre une meilleure sensibilité. Le compteur lui-même est, par contre, plus fragile, et il faut prendre garde de ne pas détériorer la fenêtre.

\section{TABLEAU I}

VALEURS MOYENNES DES ÉNERGIES MOYENNES NÉCESSAIRES POUR FORMER UNE PAIRE D'IONS DANS DIFFÉRENTS GAZ POUR LES PARTICULES $\alpha$ ET LES ÉLECTRONS [S2]

\begin{tabular}{|c|c|c|}
\hline \multirow{2}{*}{$\mathrm{Gaz}$} & \multicolumn{2}{|c|}{$\begin{array}{l}\text { Energie moyenne pour former } \\
\text { une paire d'ions }(\mathrm{eV})\end{array}$} \\
\hline & Electrons & Particules $\alpha$ \\
\hline $\begin{array}{l}\mathrm{H}_{2} \\
\mathrm{He} \\
\mathrm{N}_{2} \\
\mathrm{O}_{2} \\
\mathrm{Ar} \\
\text { air } \\
\mathrm{CO}_{2} \\
\mathrm{CH}_{4} \\
\mathrm{C}_{2} \mathrm{H}_{2} \\
\mathrm{C}_{2} \mathrm{H}_{4}\end{array}$ & $\begin{array}{l}36,6 \pm 0,3 \\
41,5 \pm 0,4 \\
34,6 \pm 0,3 \\
30,8 \pm 0,3 \\
26,2 \pm 0,2 \\
33,73 \pm 0,15 \\
32,9 \pm 0,3 \\
27,3 \pm 0,3 \\
25,7 \pm 0,4 \\
26,3 \pm 0,3\end{array}$ & $\begin{array}{l}36,2 \pm 0,2 \\
36,39 \pm 0,04 \\
32,3 \pm 0,1 \\
26,3 \pm 0,1 \\
34,98 \pm 0,05 \\
34,1 \pm 0,1 \\
29,1 \pm 0,2 \\
27,5 \\
28,03 \pm 0,05\end{array}$ \\
\hline
\end{tabular}

TABLEAU II

INPLUENCE DES IMPURETÉS SUR LA VALEUR DE L'ÉNERGIB MOYENNE NÉCESSAIRB POUR FORMER UNE PAIRE D'IONS DANS LES GAZ NOBLES [S3]

\begin{tabular}{|c|c|}
\hline $\mathrm{Gaz}$ & $\begin{array}{l}\text { Energie moyenne pour former } \\
\text { une paire d'ions }(\mathrm{eV})\end{array}$ \\
\hline $\begin{array}{l}\mathrm{He} \\
\mathrm{He} 99,87 \%-\mathrm{Ar}\end{array}$ & $\begin{array}{l}41,3 \\
29,7\end{array}$ \\
\hline $\begin{array}{c}\mathrm{Ne} \\
\mathrm{Ne} 99,88 \% \text { - Ar } \quad 0,12 \%\end{array}$ & $\begin{array}{l}36,3 \\
26,1\end{array}$ \\
\hline Ar $\begin{array}{c}\text { Ar } \\
99,8 \%\end{array}-\mathrm{C}_{2} \mathrm{H}_{4} \quad 0,2 \%$ & $\begin{array}{r}26,4 \\
21\end{array}$ \\
\hline
\end{tabular}




\section{BIBLIOGRAPHIE}

[43] BeHn Riggs, F. New design for a gas flow proportional counter. Rev. Sci. Instr. 34 (1963) 392-395.

[44] Alberici-Quaranta, A. Righini, B., Prodi, V. and Rimondi, O. On a $2 \pi$ gas-flow counter for low-level counting. Nucl. Inst. and Metbods 44 (1961) 13-23.

[45] KIEFer, H. et Maushart, R. Large-area flow counters for radiation measurements. Nucleonics, 19, $\mathrm{n}^{\circ}$ 12 (196I) $5 \mathrm{I}-54$.

[46] Fessler, H., Kiefer, H. Maushart, R. KFK-53 Karlsruhe (avril 1961) I I-153.

[47] Fessler, H. Kiefer, H., Maushart, R. KFK-37 Karlsruhe (octobre 1960) 324-326.

[48] Simpson, J.A. A precision alpha-proportional counter. Rev. of Scient. Instr. 18 (1947) 884-893.

[49] INTERTECHNIQUE - Département d'électronique. Ensemble de comptage de faibles activités alpha et béta, type RA is (1965).

[so] Cockcroft, A.L., Curran, S.C. The elimination of the end effects in counters. Rev. Sci. Instr. 22 (1951) 37-42.

[sI] Norman, R.J. A proportional counter with grid control. Aust. J. Physics 8 (1955) 419-424.

[52] Physical aspects of irradiation - Recommendations of the International Commission on Radiological Units and Measurements Handbook 85, U,S, Department of Commerce (1964).

[53] Siegbahn, K. Alpha, beta, and gamma spectrometry, North-Holland Publishing Company, Amsterdam (1965).

[54] MrLton, C.E., Hurst, G.S., Bortner, T.E. Ionization produced by s $\mathrm{MeV} \alpha$ particles in argon mixtures Phys. Rev. (1954) 643-645.

[ss] Miller, D.G., and LeBceuf, M.B. Effects of high beta backgrounds on precision alphacounting. Nucleonics $11, \mathrm{n}^{\circ} 4$ (1953) 28-31.

[56] Nirsson, G., and Anansson, G. Proportional flow counter with high-humidity gas. Nucleonics $13, \mathrm{n}^{\circ} 2$ (1955) 38-39.

[57] Handbook of Chemistry and Pbysics - $45^{\mathrm{e}}$ édition r964-1965. The chemical Rubber and Co, Cleveland - Ohio.

[58] Doввs, M.F. The use of a windowless gas-flow counter for detecting weak $\beta$ emitters on paper chromotograms. $J$. of cbromatog. 15 (1964) 29-38.

[s9] Bosh, J. Nemvuth, R. Krenz, L. German patents, 163, 989, Feb. 27 (1964).

[6o] Laboratoire Pr Berthold. Compteur à grande surface. Notice tecbnique - Wildbad (1965).

[6r] FrIeseKE - HoEpFNER - GMBH. Large area PH 407 P Methane Flow Counter. Erlangen $(1965)$.

[62] ElLo, J.G. Early detection of contamination by the use of gas proportional monitors. Health physics $9(1963) 653-658$.

[63] Williamson, C.L., Boujot, J.P., Picard, J. Tables of range and stopping power of chemical elements for charged particules of o,s to $500 \mathrm{MeV}$ - Rapport CEA R 3042 (1966).

[64] WeLler, Richard. I. Low level radioactive contamination " The Natural Radiation Environment ". Cbicago University of Cbicago Press (1964) 567-576.

[6s] Laboratoire Pr Berthold. Moniteur atmosphérique de plutonium. Notice technique. Wildbad (1965).

[66] Krenz, L. Wersse, L. An exemple for the measurement of River water low activities with methane flow counter. Mitteilungabl. Strablungsmessgereate n 6-8-9 (octobre 1961).

[67] Schmid, H. Large area methane flow counter for contamination measurement of radioactive waste water. Kernenergie 8 (1963) 286-295.

[68] Quirk, E.J.M. A monitor for low level $\beta$ radioactivity in reactor cooling water. Health Pbysics, 12 (1966) 1333-1346.

[69] Frieseke - HoEpFNER - GMBH. Fahrbaren Kontamination Monitoren. Erlangen (1965).

[70] Costrell, L. Portable $2 \pi$ flow counter for the detection of $\alpha$ and $\beta$ particles in smear samples. Healtb Pbysics 9 ( 1963$) 643$. 


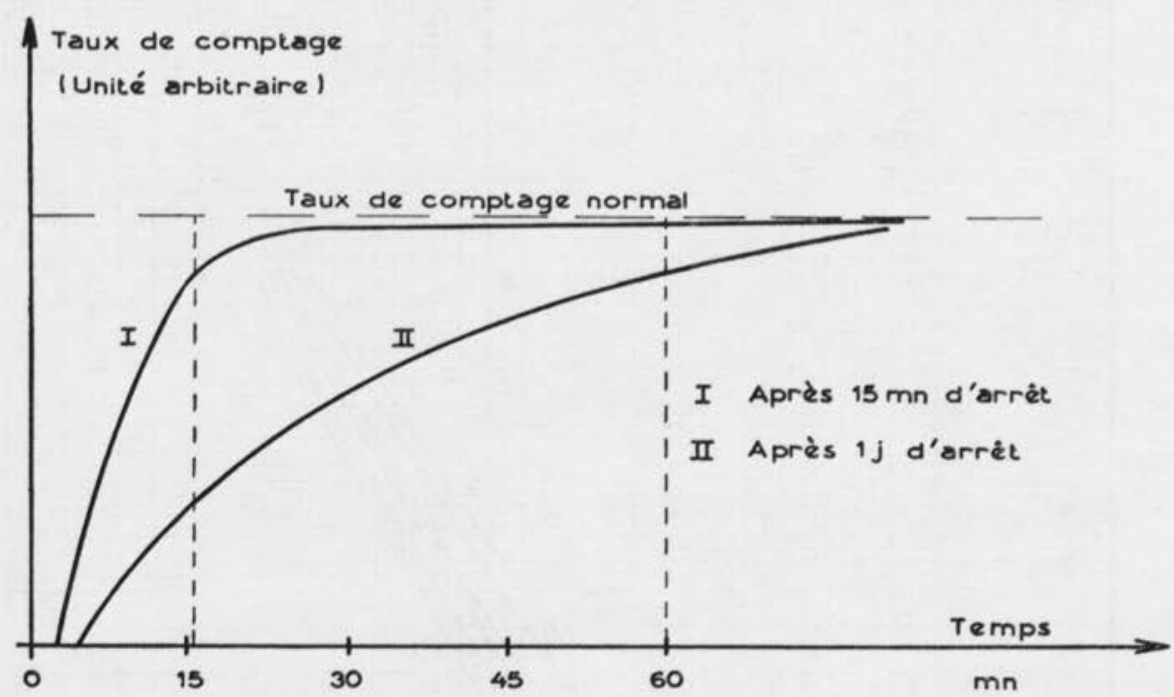

Fıg. 16. - Délai de restitution des caractéristiques normales d'un compteur à circulation de méthane Frieseke et Hœpfner type $407 \mathrm{H}$ après un arrêt de la circulation gazeuse.

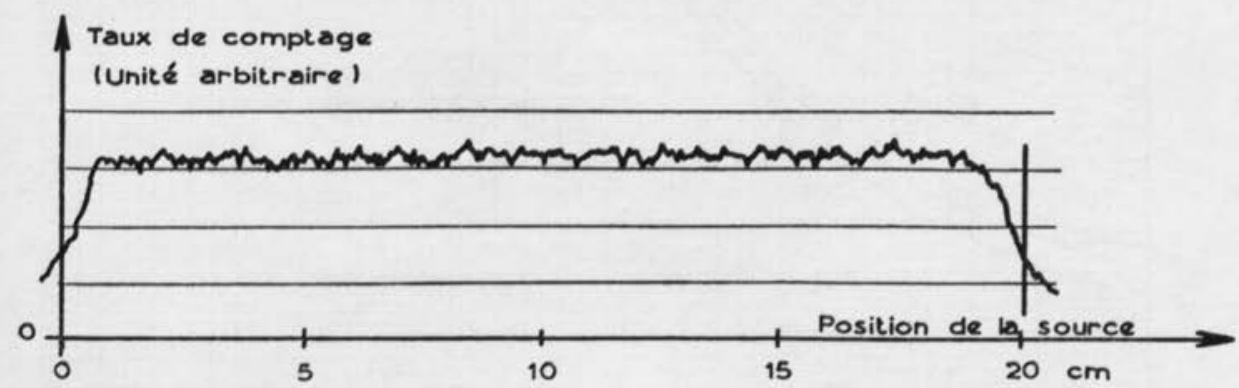

FIG. 17. - Sensibilité relative d'un compteur à circulation de méthane Berthold type LB 6301 (fenêtre en mylar aluminisé d'épaisseur $0,9 \mathrm{mg} / \mathrm{cm}^{2}$ ) 


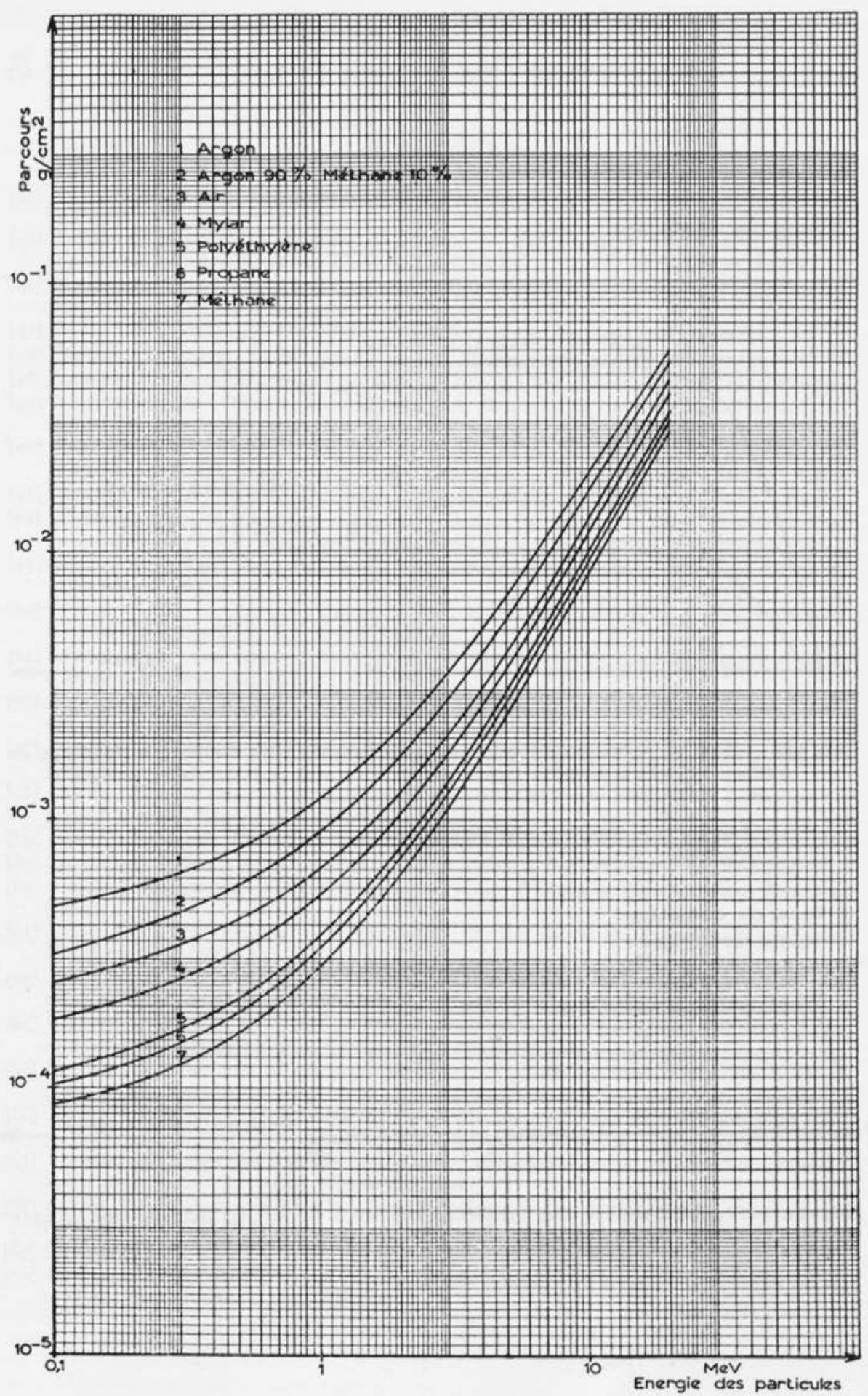

FIG. 18 a. - Parcours des particules alpha. 


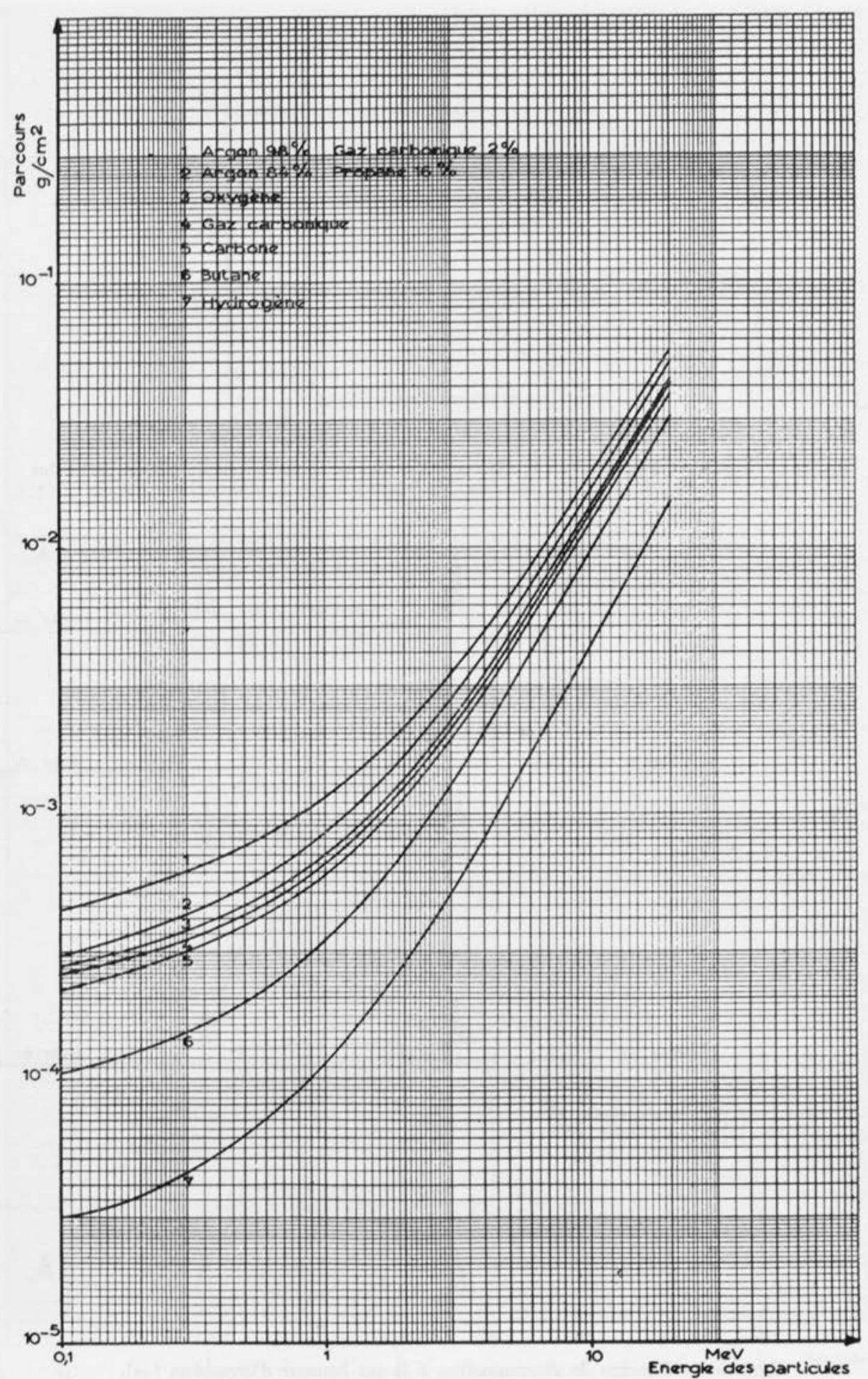

FIG. 18 b. - Parcours des particules alpha. 


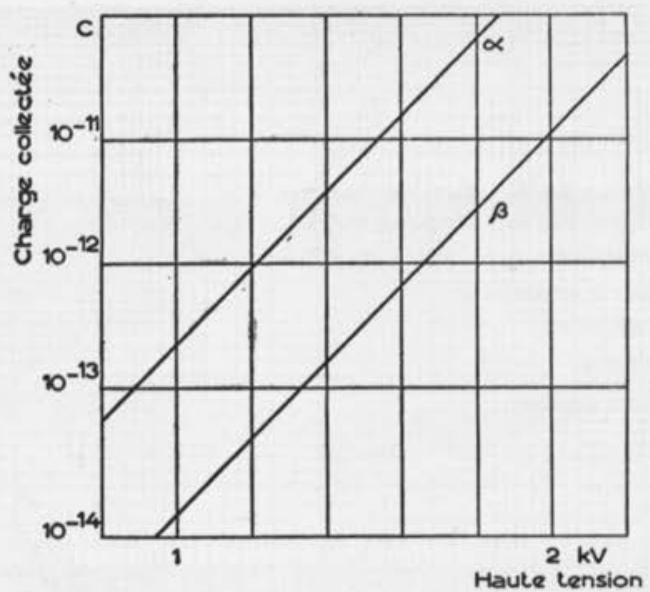

FIG. 19. - Valeurs maximales des charges collectées en fonction de la haute tension pour des particules $\alpha$ de $5 \mathrm{MeV}$ et des électrons de $2 \mathrm{MeV}$ (Compteur Intertechnique $\mathrm{S}_{35}$ )
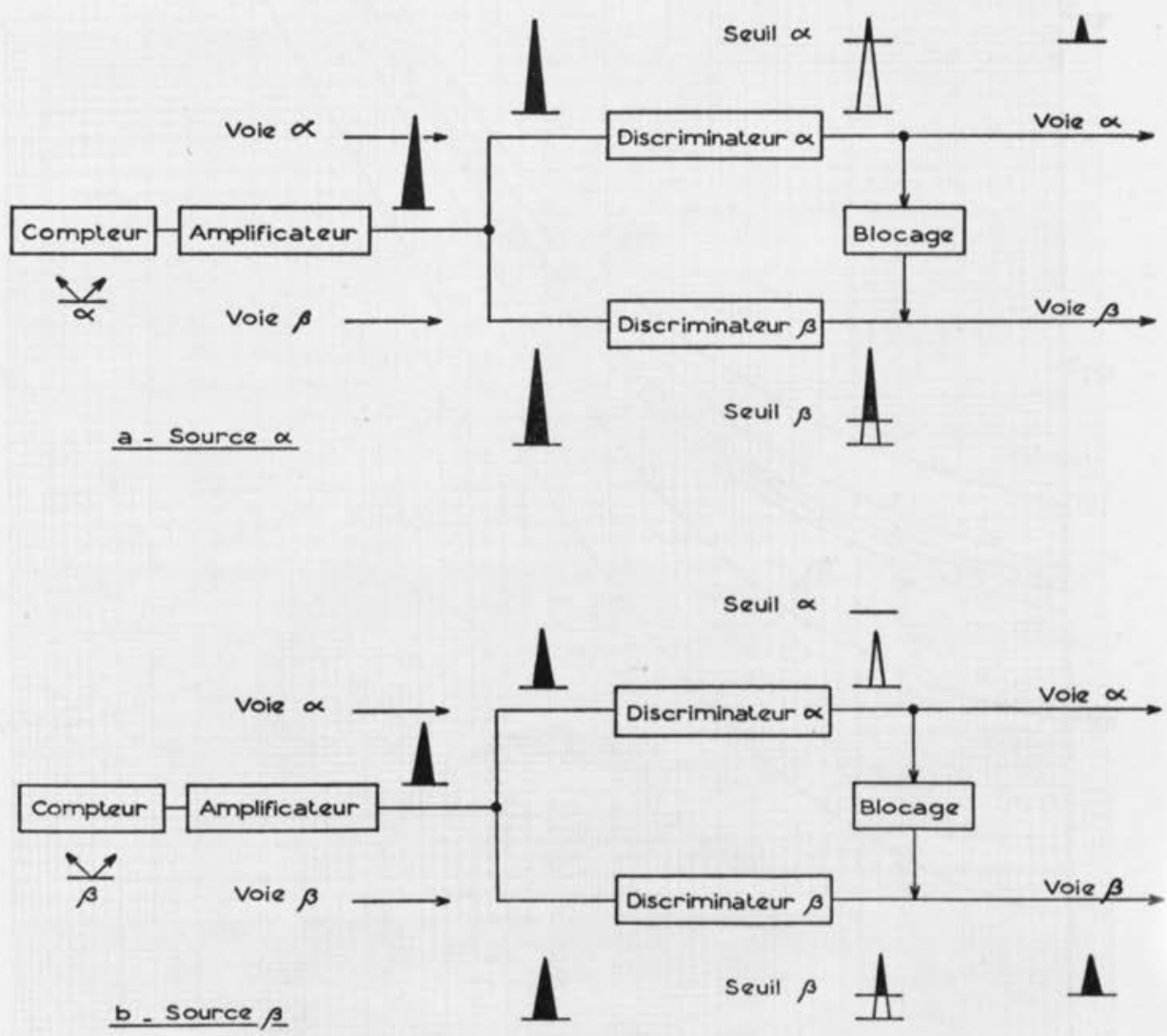

FIG. 20. - Principe de discrimination $\alpha \beta$ par hauteur d'impulsion [49]. 


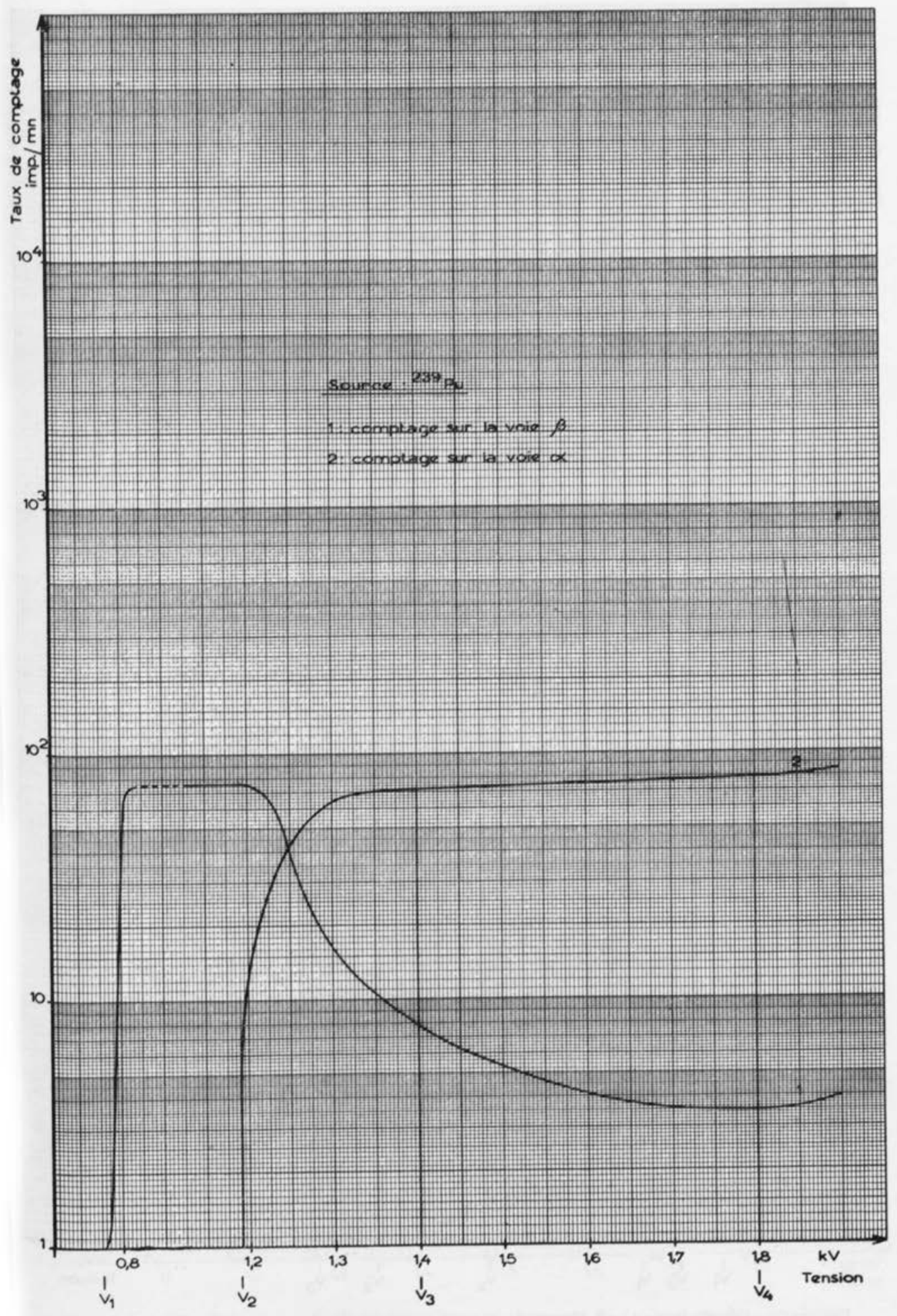

Fig. 21. - Description $\alpha \beta$ électronique avec un compteur de $135 \mathrm{~mm}$ de diamètre Intertechnique type $\mathrm{S}_{35}$ - Comptage d'un émetteur $\alpha$. 

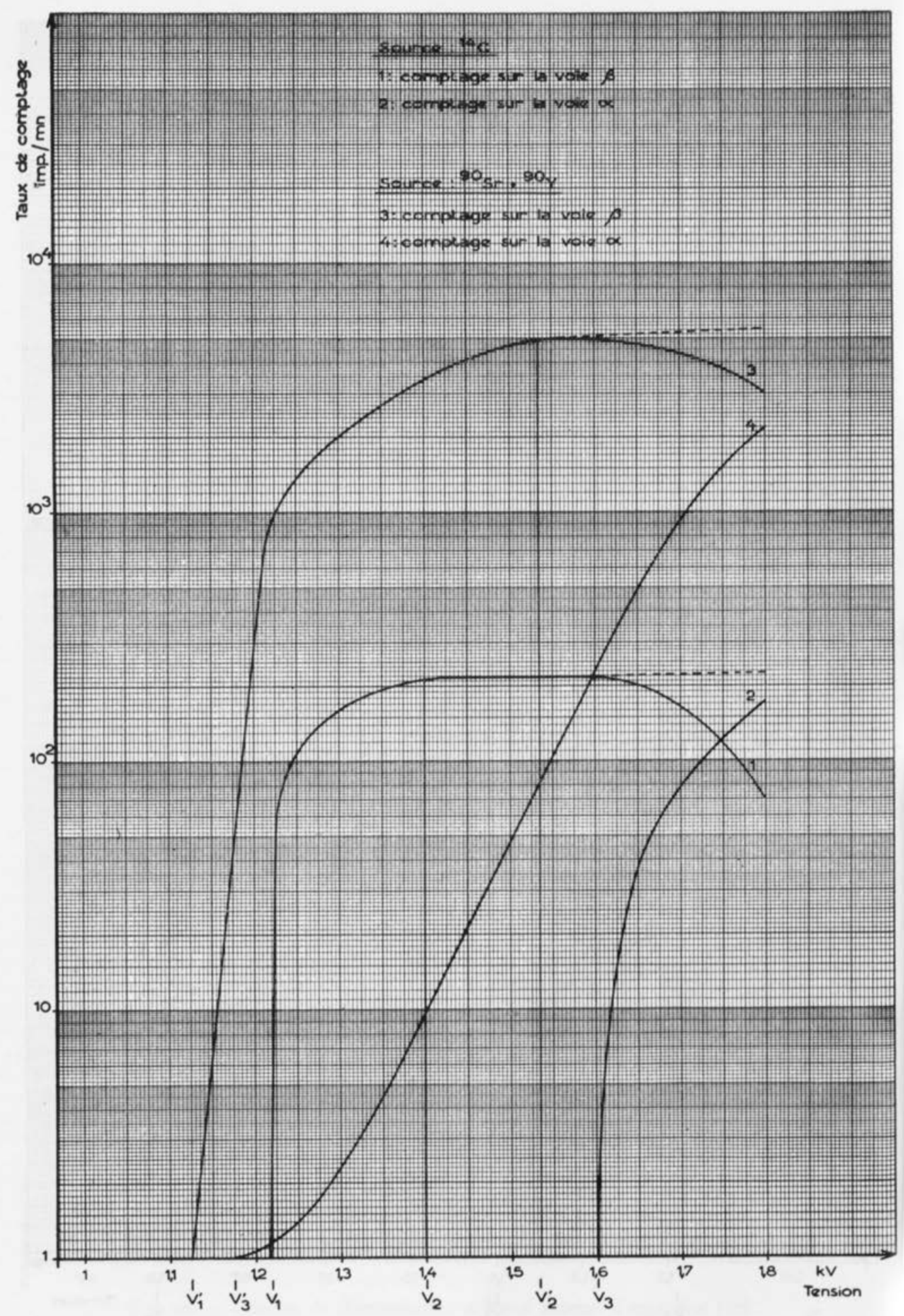

FIG. 22. - Discrimination $\alpha \beta$ électronique avec un compteur de $135 \mathrm{~mm}$ de diamètre Intertechnique type $\mathrm{S}_{35}$ - Comptage d'un émetteur $\beta$. 


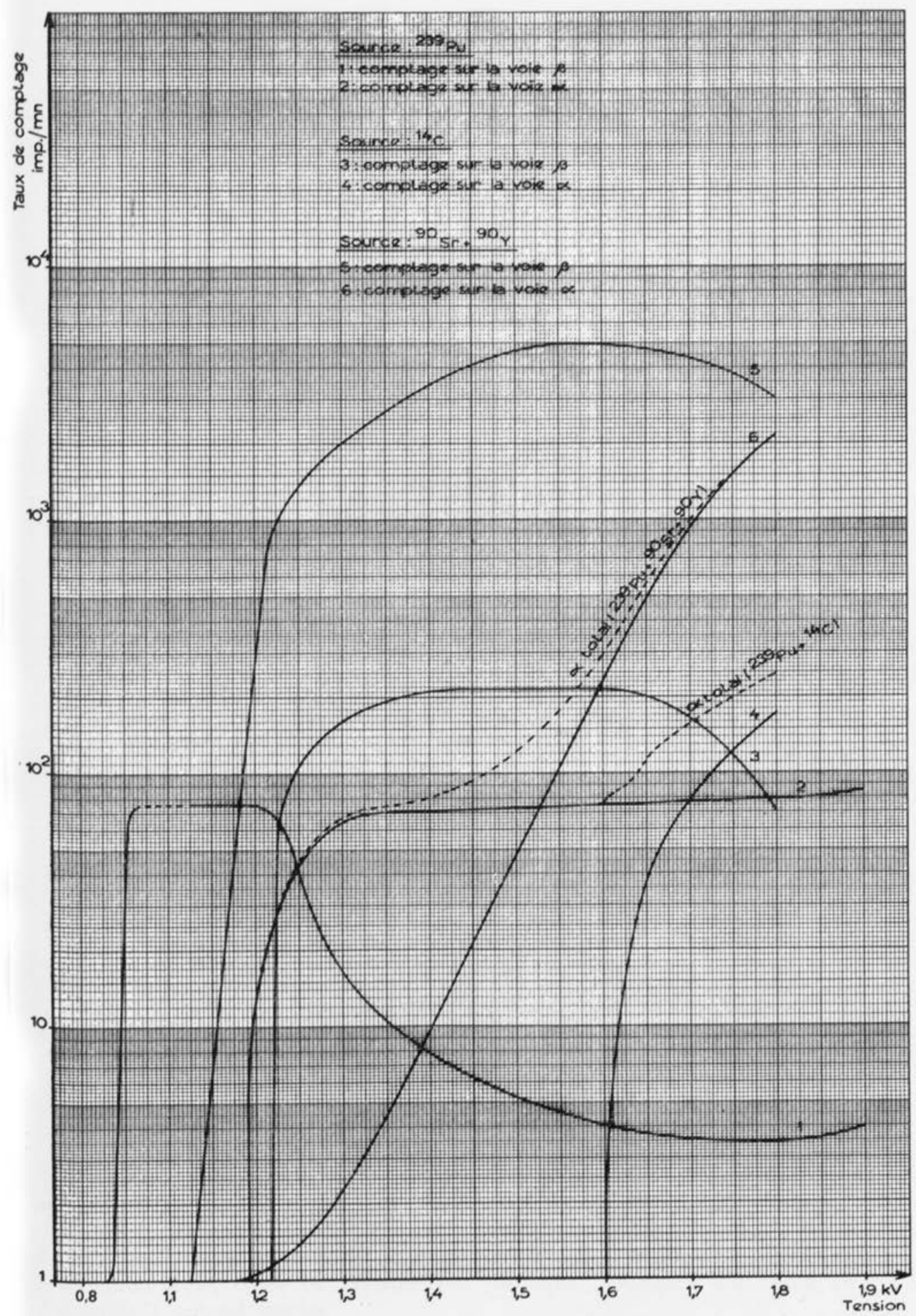

FIG. 23. - Discrimination $\alpha \beta$ électronique avec un compteur de $135 \mathrm{~mm}$ de diamètre Intertechnique type $\mathrm{S}_{35}$ - Détermination de la plage de tension de fonctionnement. 
